\title{
Correction of source-rock permeability measurements owing to slip flow and Knudsen diffusion: a method and its evaluation
}

\author{
Hui-Hai Liu ${ }^{1} \cdot$ Dan Georgi ${ }^{1} \cdot$ Jinhong Chen ${ }^{1}$ \\ Received: 4 May 2017 / Published online: 2 December 2017 \\ (c) The Author(s) 2017. This article is an open access publication
}

\begin{abstract}
Source-rock permeability is a key parameter that controls the gas production rate from unconventional reservoirs. Measured source-rock permeability in the laboratory, however, is not an intrinsic property of a rock sample, but depends on pore pressure and temperature as a result of the relative importance of slip flow and diffusion in gas flow in lowpermeability media. To estimate the intrinsic permeability which is required to determine effective permeability values for the reservoir conditions, this study presents a simple approach to correct the laboratory permeability measurements based on the theory of gas flow in a micro/nano-tube that includes effects of viscous flow, slip flow and Knudsen diffusion under different pore pressure and temperature conditions. The approach has been verified using published shale laboratory data. The "corrected" (or intrinsic) permeability is considerably smaller than the measured permeability. A larger measured permeability generally corresponds to a smaller relative difference between measured and corrected permeability values. A plot based on our approach is presented to describe the relationships between measured and corrected permeability for typical Gas Research Institute permeability test conditions. The developed approach also allows estimating the effective permeability in reservoir conditions from a laboratory permeability measurement.
\end{abstract}

Keywords Shale gas · Permeability · Knudsen diffusion - Unconventional reservoir - Source rock

\section{Introduction}

Small and exceedingly small pore size is an important feature of unconventional source-rock reservoirs. Typically, organic matter (kerogen), with pore sizes of nanometer scale (500 $\mathrm{nm}$ or smaller), is embedded within the inorganic constituents with pore sizes ranging from 10 to $100 \mu \mathrm{m}$ (Darabi et al. 2012). The small pore sizes make it very challenging to measure rock properties related to flow processes in source rocks, yet these properties are important for characterizing source-rock reservoirs and modeling the flow processes.

Source-rock matrix permeability is a key parameter to determine gas production rates from source-rock reservoirs (Ozkan et al. 2011). Because gas flow in these reservoirs is

Hui-Hai Liu

huihai.liu@aramcoservices.com

1 Aramco Research Centers - Houston, Aramco Services Company, Houston, TX 77084, USA

Edited by Yan-Hua Sun associated with slip flow, Knudsen diffusion and other mechanisms, the rock matrix permeability, unlike that for conventional rock types, are not an intrinsic rock property, but depend on gas pressure and temperature (Civan 2010; Darabi et al. 2012). Thus, laboratory permeability measurements should not be directly used for reservoir modeling studies without correction when reservoir conditions (including pressure and temperature) are considerably different from the test conditions under which laboratory measurements are made. To correct the laboratory permeability measurements, we need to know, for a given rock sample, the relationship between measured permeability and pore pressure that determines the relative importance of the combination of slip flow and Knudsen diffusion. Note that in this paper, we only discuss cases in which pore pressure may change, but effective stress imposed on a core sample remains unchanged. Thus, mechanical deformation processes do not come into play in the discussion in this paper.

There are currently two approaches to measure the porepressure dependence of gas permeability in the laboratory. The first one is to simply perform a number of pulse-decay 
permeability tests with different gas pressures (Alnoaimi and Kovscek 2013). Then, these tests will provide gas permeability values for a number of gas pressures. The pulse-decay test setup generally consists of two gas reservoirs and a sample holder with controlled confining stress for test samples. Initially, the system is in equilibrium with a given gas pressure. A small pressure pulse is then introduced into the upstream gas reservoir, such that the pulse does not have a significant disturbance to the gas pressure in the system. The pressures at the two gas reservoirs are monitored as a function of time. The pressure evolution results are fitted using analytical solutions, with permeability being a fitting parameter. The advantage of this approach is that the test setup and data interpretation procedure are relatively simple. However, it generally takes a relatively long time to equilibrate the test system from one test pressure to the next one (Jones 1997).

The other laboratory approach to determine the pressure dependence is to first develop a formulation of gas permeability as a function of gas pressure and then estimate values for parameters in the formulation by numerically matching the relevant test results under different gas pressure conditions (Civan et al. 2012). Test results are generally from pulse-decay tests in which the pressure pulse is not limited to a small one because the numerical model is flexible enough to incorporate the pulse disturbance to the system. However, non-uniqueness of parameter estimation is always a problem for parameter estimations with the inverse modeling.

In addition to the above two laboratory measurement approaches, the permeability correction may be made with a given relationship between intrinsic permeability, measured permeability, and laboratory test conditions. This approach has an obvious advantage over the two laboratory measurement approaches discussed above because it allows for estimating intrinsic permeability and the relationship (between measured permeability and pore pressure) from one permeability measurement for a given pore pressure. Note that for the laboratory approaches, the relationship of pore-pressure dependency requires permeability measurements at multiple pore pressures that are very time-consuming. Furthermore, a relationship between intrinsic permeability and laboratory test conditions can also be used to relate laboratory permeability measurements to reservoir conditions in which pore pressure (and temperature) is generally different from those used in the laboratory and changes with time and location.

The challenge related to the last approach is the determination of a practically useful relationship between measured permeability, laboratory test conditions and intrinsic permeability. This is largely because of the complexity of pore structure in a porous medium. For example, gas flow processes in a porous medium may be subject to different flow regimes within different pores; diffusion is important only within small pores for a given pore pressure. On the other hand, the relationships between measured permeability and intrinsic permeability have been well developed for micro/nano-tubes partially as a result of the simplicity of their flow-path geometries (Beskok and Karniadakis 1999). Then efforts have been made in using the relationships for micro/nano-tubes to approximate those for porous media (Civan 2010; Ziarani and Aguilera 2012; Singh et al. 2014). However, the usefulness of these approximations is not totally clear because comparisons between results calculated with these approximations and laboratory measurements are very rare in the literature.

In this paper, we present an approximate relationship (between measured permeability, laboratory test conditions and intrinsic permeability) that is also based on the corresponding relationship for micro/nano-tubes and then validate the relationship with several carefully selected data sets for carbonate source rocks published in the literature. (We focus on carbonate source rock herein because it is an important source-rock type.) The workflow to apply the relationship to the correction of laboratory permeability measurements is discussed as well. The major purpose of this work is to provide a practical and yet simple way to determine the source-rock intrinsic permeability by correcting the corresponding laboratory permeability measurement. It may be important to emphasize that this work differs from the previous studies at least in the following aspects. The paper systematically demonstrates the usefulness of a simple approach for practical application that has a potential to become a standard method for permeability-measurement correction in the laboratory. It also clearly shows, by comparing the calculation results with the literature, that a source-rock sample can be represented as micro/nano-tubes (with a size estimated from permeability and porosity) for the permeability-correction purpose at least for certain types of source rocks, while previous studies have mainly considered the treatment as a conceptual assumption.

\section{Theoretical background}

The pore-pressure dependency of matrix permeability is attributable to the effect of slip flow and diffusion on the gas flow. It is worthwhile to revisit some basic concepts of gas diffusion to set the stage for further discussion. Diffusion is a process of net movement of a substance from a high-concentration region to a low-concentration region. The magnitude of the diffusive flux is proportional to concentration gradient multiplied by a diffusion coefficient. Gas diffusion is really a result of thermal motion of gas 
molecules. Based on the classic statistical physics, the gas diffusion coefficient is given by (Singh et al. 2014)

$D=\frac{1}{3} \lambda u$

where $D$ is the diffusion coefficient; $\lambda$ is the mean free path; and $u$ is the averaged magnitude of thermal motion speed. The latter two parameters are given by (Civan 2010; Ziarani and Aguilera 2012; Singh et al. 2014; Liu and Cai 2014)

$\lambda=\frac{k_{\mathrm{B}} T}{\sqrt{2} \pi d_{\mathrm{c}}^{2} p}$

$u=\sqrt{\frac{8 k_{\mathrm{B}} T}{\pi M}}$

where $k_{\mathrm{B}}$ is the Boltzmann constant $\left(1.3805 \times 10^{-23} \mathrm{~J} / \mathrm{K}\right)$; $T$ is the temperature, $\mathrm{K} ; p$ is the pressure, $\mathrm{Pa} ; d_{\mathrm{c}}(\mathrm{m})$ is the collision diameter for gas that is $0.42 \mathrm{~nm}$ for methane and $0.358 \mathrm{~nm}$ for $\mathrm{N}_{2}$ that is often used as a working fluid to measure permeability in laboratory; and $M$ is the gas molecular weight, $\mathrm{kg} / \mathrm{mol}$. The mean free path is the average distance for a molecule to travel before it hits another molecule. When the pore size is very small, the mean free path cannot be used to characterize the gas diffusion process, because gas molecule movement will be constrained by the pore walls. When the mean free path is larger than the pore diameter, the former in Eq. (1) should be replaced by the latter. In this case, the gas diffusion coefficient becomes (Singh et al. 2014)

$D_{\mathrm{n}}=\frac{1}{3} d u$

This diffusion coefficient is called the Knudsen diffusion coefficient, and the corresponding process is called Knudsen diffusion. The parameter $d$ in the above equation is the pore diameter.

As discussed in Ziarani and Aguilera (2012) and references cited therein, gas flow in a capillary tube can be classified into four regimes based on the dimensionless number (Knudsen number) defined by

$K n=\frac{\lambda}{r}$

where $r$ is the pore radius.

The first flow regime corresponds to $K n<0.01$ and is called Darcy flow or viscous-flow regime (Ziarani and Aguilera 2012). Gas can be described as a continuum, and the dominant mechanism is viscous flow. The fluid velocity at the solid surface is zero. Thus, the gas flow can be adequately modeled with Darcy's law.

The slip-flow regime corresponds to $0.01<K n<0.1$ (Ziarani and Aguilera 2012). Slip flow occurs when the gas molecules experience slipping at the solid surface. In this case, gas flow can still be dealt with within the context of continuum mechanics. Conventional flow equations can be used with certain modifications associated with slip-flow boundary condition.

Transition-flow regime is defined for $0.1<K n<10$ (Ziarani and Aguilera 2012). In this regime, both slip-flow (continuum) and diffusion are important flow mechanisms. It is very likely that the conventional form of Darcy's law is not valid or adequate and, thus, reservoir simulations based on it may not be reliable unless the permeability in Darcy's law is treated as an apparent or effective parameter.

The last flow regime is the Knudsen diffusion (or flow) regime with $K n>10$. For this regime, continuum mechanics breaks down and the gas flow process is dominated by Knudsen diffusion. Figure 1 shows a comparison between normal diffusion and Knudsen diffusion. In a normal diffusion process, the molecule movement is largely controlled by the collisions among molecules themselves, because the mean free path is much smaller than the pore size. For Knudsen diffusion, the diffusion process is largely controlled by collisions between molecules and pore walls; collisions among molecules are rare.

There are two approaches available in the literature to calculate gas flow process in micro-tubes. One approach is based on a fundamental assumption that the total gas flow rate through a micro-tube is a summation of the following two components (Roy et al. 2003; Singh et al. 2014). One corresponds to viscous flow (determined with classic pipe flow formulations) and the other one to Knudsen diffusion (determined with diffusion coefficient given in Eq. 4). According to Roy et al. (2003), this treatment seems to be adequate for practical applications.
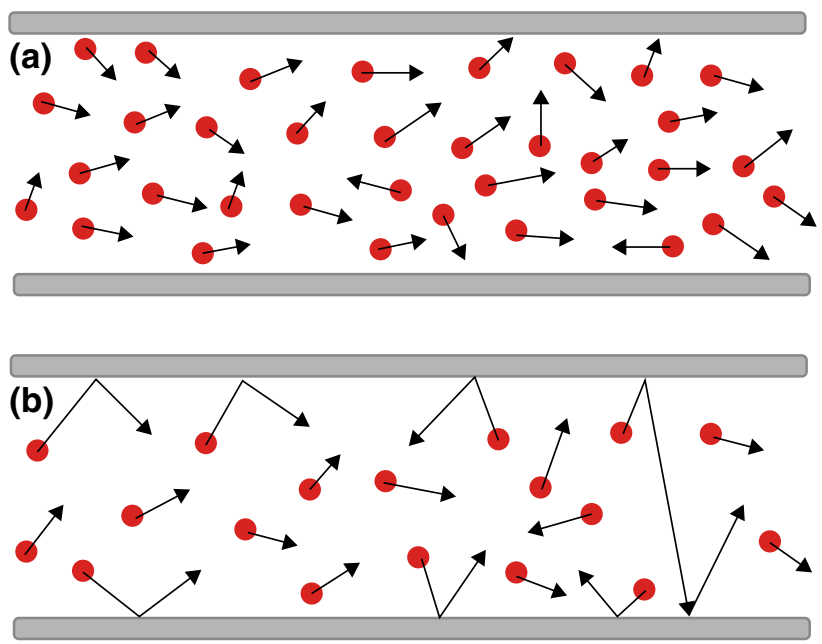

Fig. 1 A sketch of normal gas diffusion (a) and Knudsen diffusion (b) in a pore 
The other approach was developed by Beskok and Karniadakis (1999). They wrote that the gas flow rate through a micro-tube is the rate owing to viscous flow multiplied by a correction factor that is a function of Knudsen number and given by

$f_{\mathrm{c}}=(1+\alpha K n)\left[1+\frac{4 K n}{1-b K n}\right]$

where $b$ is a constant and equal to -1 , and $\alpha$ is the dimensionless rarefaction coefficient. The ratio of effective (measured) rock permeability to $f_{\mathrm{c}}$ is the intrinsic (corrected) permeability. An approximation formulation to calculate $\alpha$ is given by Civan (2010):

$\frac{\alpha_{0}}{\alpha}-1=\frac{A}{K n^{B}}$

where $\alpha_{0}=64 /(15 \pi), \quad A=0.1780$ and $B=0.4348$. Equation (6) is empirical in nature, but extensively validated against other theoretical methods such as direct simulation of Monte Carlo (DSMC), linearized Boltzmann solution (LBS), and experimental results.

Figure 2 shows the correction factor as a function of Knudsen number calculated with Eq. (6). For Darcy flow, the correction factor is equal to one. Clearly, for a relatively large $K n$, the use of Darcy's law without modification can result in significant errors. Also note that the formulation of Beskok and Karniadakis (1999) mathematically employs pressure gradient as a driving force. Since the gas pressure gradient is closely related to the concentration (or density) gradient, the formulation is still valid for diffusion-dominated regimes, as discussed in Ziarani and Aguilera (2012). Also note that the correction factor is related to temperature at a given pore pressure, because the mean free length is a function of temperature as well.

Efforts have been made to develop gas flux formulations for porous media based on the previous results for

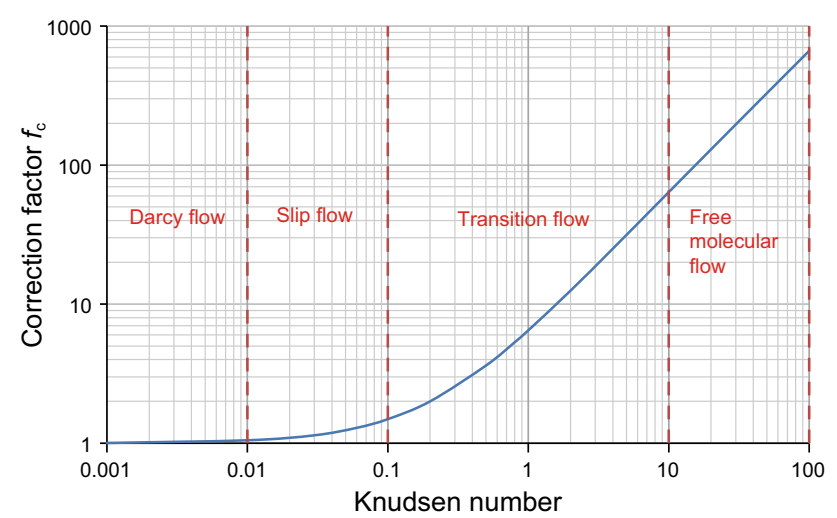

Fig. 2 Correction factor as a function of Knudsen number and for different flow regimes (after Ziarani and Aguilera 2012) micro/nano-tubes (Civan 2010; Javadpour 2009). The main strategy is to estimate a representative pore size for a porous medium to calculate the Knudsen number. If the porous medium can be represented by a group of identical capillary tubes with radius $r$, the (intrinsic) permeability $k$ will be given by (Liu 2017, Chapter 1)

$k=\frac{r^{2}}{8} \phi$

where $\phi$ is the porosity of the porous medium.

In a porous medium, the intrinsic permeability is largely controlled by the pore-throat radius $r$. A rigorous relation between the intrinsic permeability and a representative pore-throat radius $r$ is

$k=\frac{r^{2}}{8} \phi \frac{L_{\mathrm{th}}}{L_{\mathrm{f}}} \frac{L_{\mathrm{f}}}{L_{\mathrm{x}}}$

where $L_{\mathrm{f}}$ is the total length of the flow path from the inlet to the outlet of a rock sample; $L_{\mathrm{th}}$ is the total length occupied by pore throats along the flow path; and $L_{\mathrm{x}}$ is the distance between the inlet and the outlet of the rock sample. Obviously, we have $L_{\mathrm{th}} / L_{\mathrm{f}} \leq 1$ and $L_{\mathrm{f}} / L_{\mathrm{x}} \geq 1$; their values likely depend on specific formations.

For simplicity, we here assume

$\frac{L_{\mathrm{th}}}{L_{\mathrm{f}}} \frac{L_{\mathrm{f}}}{L_{\mathrm{x}}} \approx 1$

The validity of this approximation will be demonstrated by the fact that the pore-pressure dependency of effective permeability, predicted based on Eq. (10), is reasonable compared with the data. The prediction will be discussed later in the next section. In this case, Eq. (9) is reduced to Eq. (8). Thus, the representative pore-throat radius can be estimated by

$r=\sqrt{\frac{8 k}{\phi}}$

It is important to emphasize that $k$ here is the intrinsic permeability.

In summary, once the intrinsic rock permeability $k$, working fluid, and laboratory test conditions ( $T$ and $p$ ) are given, one can calculate the Knudsen coefficient $K n$ using Eqs. (2), (11) and (5), and then calculate $f_{\mathrm{c}}$ from Eq. (6). The intrinsic permeability multiplied by $f_{\mathrm{c}}$ will be the effective permeability value measured under the laboratory test conditions. Accordingly, for the given laboratory measurement results, one can also estimate the intrinsic permeability value through inverse calculations. This will be further discussed in Sect. 4. Note that in this paper, we use terms "intrinsic permeability" and "corrected permeability" interchangeably. 


\section{Verification with laboratory measurement data}

In Sect. 2, an approach is proposed to estimate the porepressure dependency of effective permeability. Since it is based on the theory for gas flow in a micro/nano-tube and an approximate way to determine the representative porethroat radius from the intrinsic permeability, the usefulness of the approach needs to be verified before we apply it to permeability measurements.

The verification uses the following procedure. For a given data set consisting of measured rock properties as a function of pore pressure, we estimate the intrinsic permeability from the permeability measurement with the highest pore pressure because that measurement is closest to the intrinsic permeability value. (Fig. 8 to be discussed later gives the detailed procedure to estimate the intrinsic permeability.) We then use the intrinsic value to predict the effective permeability measurements at different pore pressures and compare predictions with the observed values. The data sets of measured permeability as a function of pore pressure (under the same effective stresses) are limited in the literature. In this section, we verify our approach using three data sets that were collected at room temperature (assumed to be $22{ }^{\circ} \mathrm{C}$ ) and used $\mathrm{N}_{2}$ as the working fluid.

The first data set is from Jin et al. (2015) who documented permeability measurements for some unidentified unconventional rock samples from North America. They reported results for seven samples, but we only use the five samples for which they also reported porosity values (Jin et al. 2015). The porosity is needed to estimate the porethroat radius (Eq. 11). Jin et al. (2015) measured the rock permeability using the pulse-decay method at two different pore pressures: $9 \mathrm{MPa}$ (or $1305 \mathrm{psi}$ ) and $4 \mathrm{MPa}$ (or 580 psi). We estimated the intrinsic permeability from the measurement at 1305 psi and predict the measurement at $580 \mathrm{psi}$ for each of the five rock samples.

Figure 3 shows a comparison between observed and predicted relative changes in permeability measurements, as a function of permeability measurement at a pore pressure of 1305 psi, for rock samples reported in Jin et al. (2015). Note that in this paper, we use terms "observed permeability", "measured permeability" and "effective permeability" interchangeably and subscript "eff" refers to the effective permeability. The relative change here is defined as the ratio of the difference between permeability measurements (at 580 and 1305 psi) and the permeability measurement at $1305 \mathrm{psi}$. As predicted, the relative change increases with decreasing permeability measurement at $p=1305$ psi, because a smaller “ $k_{\text {eff } @ p=1305}$ psi” corresponds to a smaller representative pore size and thus a

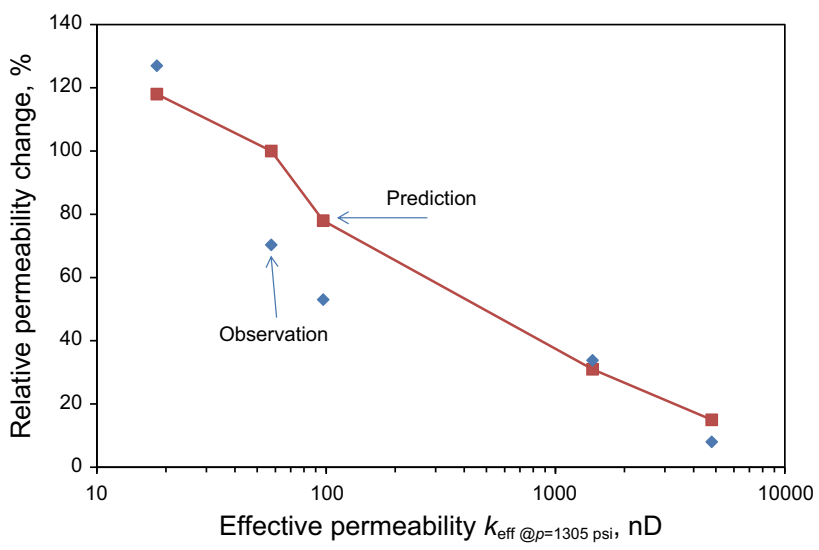

Fig. 3 Comparison between observed (blue) and predicted (red) relative changes in effective permeability measurements, as a function of permeability measurement at a pore pressure of 1305 psi, for 5 rock samples reported in Jin et al. (2015). The relative change is defined as the ratio of change in permeability measurements (from 580 to $1305 \mathrm{psi}$ ) to the permeability measurement at $1305 \mathrm{psi}$

more important contribution of Knudsen diffusion to gas flow. To further demonstrate this point, Fig. 4 shows a comparison between permeability measurements at 1305 psi and the corresponding intrinsic permeability values (estimated from the workflow shown in Fig. 8); their differences become larger for low-permeability values. Nevertheless, the relative changes in effective permeability from pore pressure of 580-1305 psi are reasonably predicted for a large range of values of “ $k_{\mathrm{eff} @ p=1305 \mathrm{psi}}$ " (Fig. 3).

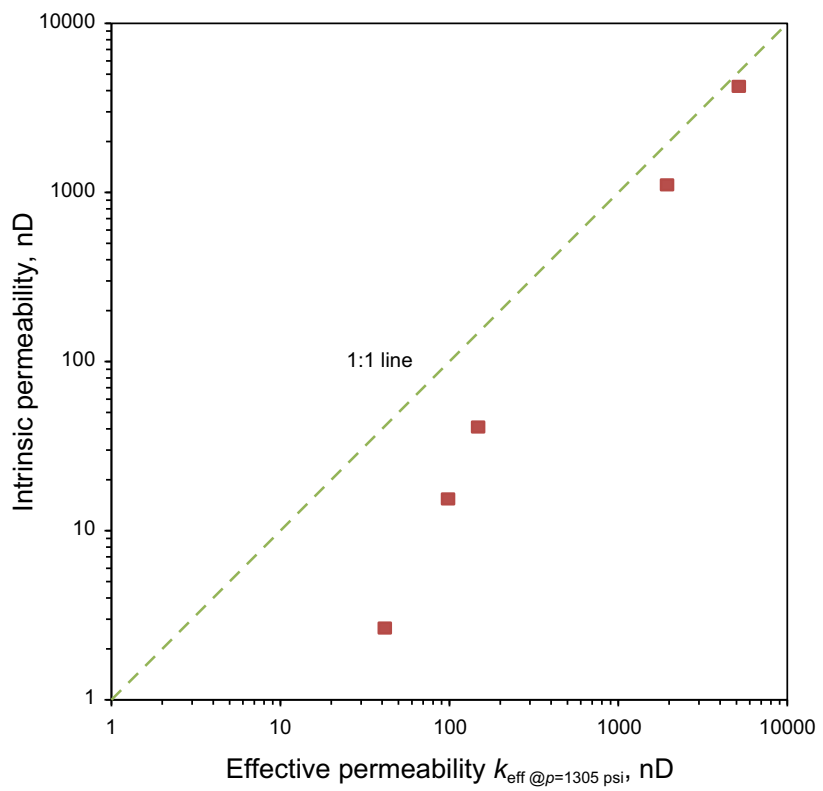

Fig. 4 Comparison between permeability measurements at 1305 psi and the corresponding corrected or intrinsic permeability values for rock samples reported in Jin et al. (2015). Their differences become larger for low-permeability values 
Alnoaimi and Kovscek (2013) reported observed permeability values as a function of pore pressure for an Eagle Ford rock sample. The measurements were taken with the pulse-decay method. The rock sample has a porosity of $6.2 \%$ and contains calcite-filled fractures. Again, we estimate the intrinsic permeability value at the highest pore pressure used in the test and predict permeability measurements at other pore pressures. Figure 5 shows the predicted and observed effective permeability values as a function of pore pressure. Although our approach seems to underestimate permeability measurements especially for relatively low pressures, our predictions should be considered reasonable given the challenges for accurately measuring source-rock permeability in the laboratory (Singh et al. 2014).

Heller et al. (2014) reported a comprehensive study of the measurements of matrix permeability of gas shales. For a given effective stress, they reported measured-permeability data points for several pore pressures $(1000,2000$, 3000 , and $4000 \mathrm{psi}$ ) and the same effective stress for two Eagle Ford samples. The porosity values for these samples are not given in Heller et al. (2014) and are assumed to be a typical value of $7 \%$. Again, we estimate the intrinsic permeability value for a given rock sample at the highest pore pressure used in the test and predict permeability measurements at other pore pressures. Figure 6 shows that reasonable comparisons between observed and predicted effective permeability values as a function of pore pressure for the two Eagle Ford rock samples reported in Heller et al. (2014) [Note that only the data of Heller et al. (2014) under the smallest confining stress are used here because they can be more accurately estimated from the relevant plots in the paper].

Our predicted effective permeability values at different pore pressures are generally consistent with observations

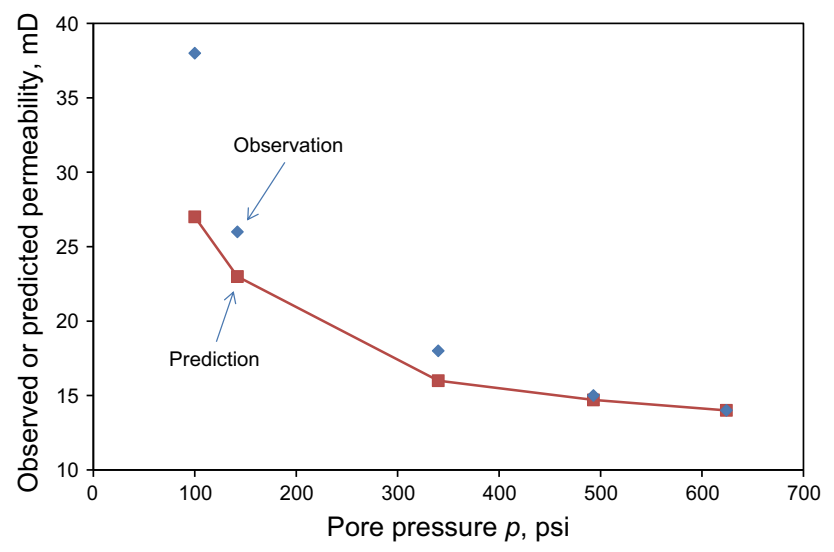

Fig. 5 Comparison between observed and predicted permeability values as a function of pore pressure, at a constant net confining stress, for an Eagle Ford rock sample reported in Alnoaimi and Kovscek (2013) from the three data sets that cover a large range of observed permeability values. This, combined with the theory, gives us confidence in the usefulness of our approach. It is also worth noting from Figs. 3, 5 and 6 that the approach is probably on the conservative side in the sense that the approach, on average, underestimates the pore-pressure dependency. It is possible that the use of a single pore size (Eq. 11) may sometimes underestimate the diffusion effect in a porous medium that has a range of pore sizes; different pores can be subject to different degrees of the diffusion effect. This needs further investigation.

To further demonstrate the importance of pore-pressure dependency in analysis of laboratory permeability measurements, Fig. 7 presents the correlation factor, calculated from Eq. (6), as a function of pore pressure and for two temperatures $\left(25\right.$ and $\left.125^{\circ} \mathrm{C}\right)$. For pore pressures larger than $400 \mathrm{psi}, f_{\mathrm{c}}$ changes very slowly with increasing pore pressure. Since the measured permeability is equal to this correction factor multiplied by the intrinsic permeability, sometimes one may think that the intrinsic permeability is obtained at around 400-500 psi for the case in Fig. 7. In that pressure range, the permeability measurements seem to be stabilized. In fact, the effective permeability still largely results from Knudsen diffusion because $f_{\mathrm{c}}$ is considerably larger than one. The intrinsic permeability corresponds to $f_{\mathrm{c}}=1$. Also note that for a given pore pressure, a higher temperature generally gives rise to a larger $f_{\mathrm{c}}$ value because it corresponds to a larger mean free path (Eq. 2).

\section{Correction of laboratory permeability measurements}

Since the theoretical formulations developed in Sect. 2 can be used to reasonably predict observed pore-pressure dependency of measured (or effective) gas permeability, an approach based on these formulations is proposed here to correct the laboratory permeability measurements. By "correction", we mean the determination of the intrinsic permeability from the effective permeability value, rock porosity, temperature, gas pressure and the type of working fluid. While the details of the workflow are given in Fig. 8, the key steps for the "correction" include the following ones.

We calculate the mean free path based on temperature, gas pressure and the type of working fluid used for measuring effective permeability in laboratory. We then assume an intrinsic permeability value and calculate the pore-throat radius from the assumed value and porosity. With the mean free path and the radius, we can calculate the Knudsen number and further calculate the correction factor $f_{\mathrm{c}}$. Multiplying the intrinsic permeability with $f_{\mathrm{c}}$ gives the predicted measured permeability for the given 

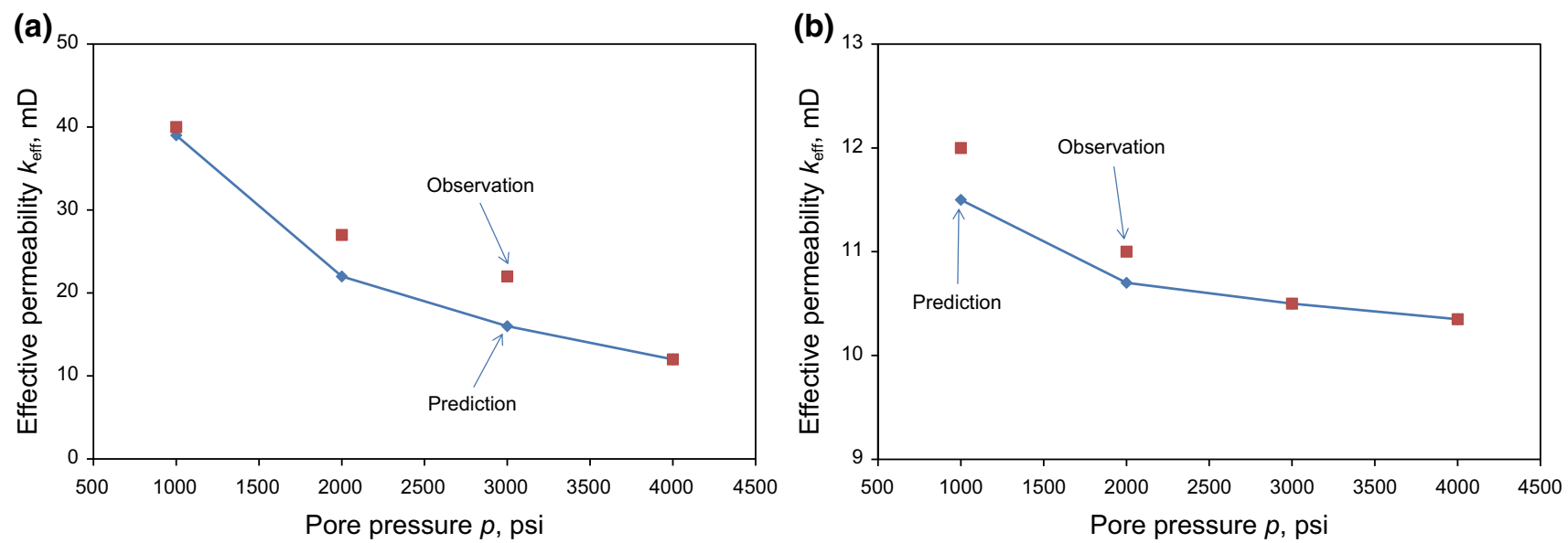

Fig. 6 Comparisons between observed and predicted effective permeability values as a function of pore pressure for the two Eagle Ford rock samples reported in Heller et al. (2014). a Eagle Ford 174. b Eagle Ford 127

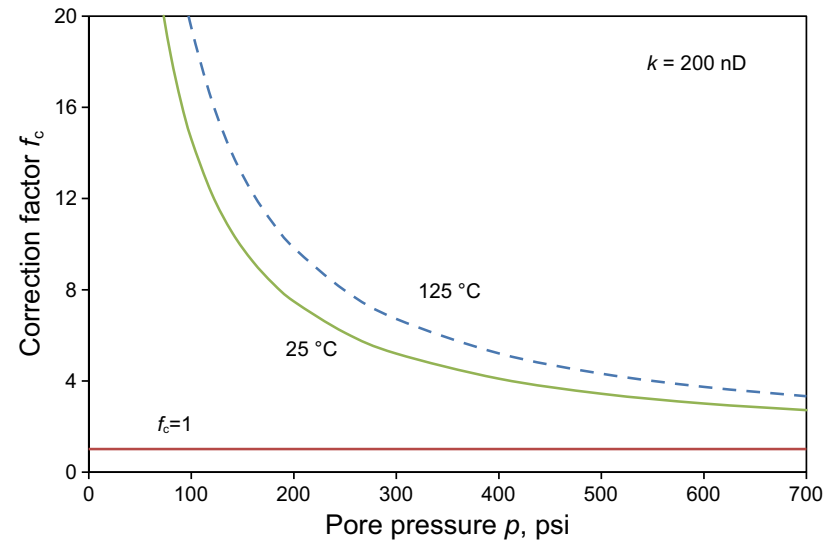

Fig. 7 Correction factor $\left(f_{\mathrm{c}}\right)$ as a function of pore pressure at different temperatures $\left(25\right.$ and $\left.125^{\circ} \mathrm{C}\right)$, calculated with Eq. (6)

pore pressure and temperature. After that, we compare the prediction with the observed gas permeability. If they are practically identical, then the assumed intrinsic permeability value can be considered correct. Otherwise, the assumed intrinsic permeability needs to be updated and the relevant calculation steps mentioned above will repeat. If the prediction is lower than the observed permeability, the assumed intrinsic permeability needs to be increased. Otherwise, it should be decreased. The secant method is used for updating intrinsic permeability here (Myron and Eli 1998).

With the above workflow, we can estimate the intrinsic or corrected permeability values from the permeability measurements. For example, Fig. 9 shows the estimated relation between the intrinsic permeability and laboratory measurements under some typical Gas Research Institute (GRI) test conditions $\left(T=22^{\circ} \mathrm{C} ; p=100-500 \mathrm{psi}\right)$. The power relation between measured permeability and porosity reported by Hakami et al. (2016) is used for calculating the curves in the figure as an example. This is because this study has focused on carbonate source-rock samples while the relationship reported by Hakami et al. (2016) was developed for a carbonate source-rock reservoir in Saudi Arabia. $\mathrm{N}_{2}$ is assumed to be the working fluid. Clearly, the measured GRI permeability, depending on the pore pressure used, can be significantly higher than the corresponding intrinsic permeability. Given the fact that the GRI method has been widely used for estimating source-rock permeability, the comparisons in Fig. 9 highlight the need to correct the laboratory permeability measurements so that they are meaningful for practical applications.

In addition to the GRI method, the other widely used laboratory technique to measure source-rock permeability is the pressure pulse-decay method (Jones 1997). Comparisons between source-rock permeability measurements with GRI and pulse-decay methods have been discussed by several groups (Cui et al. 2009; Heller et al. 2014). Some inconsistent findings have been reported, which highlight the challenges in measuring source-rock permeability and even in understanding the measurements themselves. While a detailed discussion of this issue is beyond the scope of this paper, we should at least keep the two competing mechanisms in mind when comparing the measurements from these two methods.

The first mechanism is the pore-pressure dependency of source-rock permeability, as discussed in this study. The GRI measurements are conducted generally at much lower pore pressures than those used by the pulse-decay methods. Thus, for a given intrinsic permeability, the GRI method should give a higher effective permeability. The second mechanism is related to micro-structures of rock samples under investigation. The GRI method uses rock particles with small sizes (e.g., $1 \mathrm{~mm}$ ) and therefore the measurement results are essentially not subject to the impacts of 


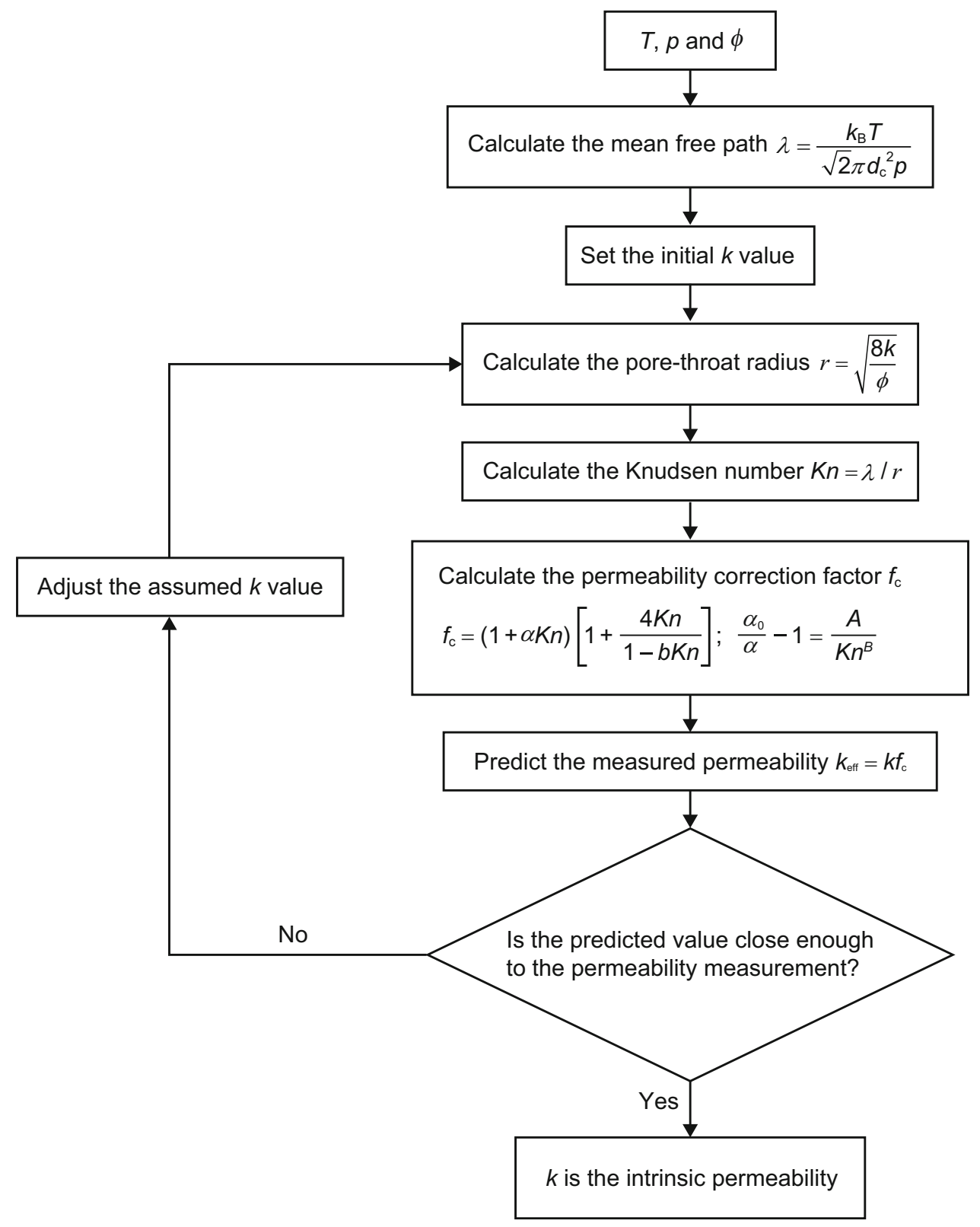

Fig. 8 Flowchart for estimating the intrinsic permeability

micro structures because these structures are at relatively large scales. At the same time, the pulse-decay permeability may mainly come from these micro-structures especially in the bedding (horizontal) direction. In other words, the GRI and pulse-decay methods may measure different types of permeability. In this case, the pulse-decay method intends to give a higher permeability measurement. Depending on which of the above two mechanisms prevails for a given test condition, the GRI permeability may or may not be higher than the pulsedecay permeability.

Finally, it is important to note that our permeabilitycorrection approach also allows estimating effective permeability under reservoir conditions and should be used in reservoir simulations. It is the effective permeability corresponding to the reservoir conditions that should be used in reservoir simulators that describe the gas flow in source rocks with Darcy's law. From a laboratory permeability measurement, we can use the methodology reported in this paper to estimate intrinsic permeability that is a constant for a given effective stress. Pore pressure and temperature are functions of both location and time in reservoirs. Then, for a given location and time, the reservoir effective permeability is equal to the intrinsic permeability multiplied by the correction factor (Eq. 6) that is a function of reservoir temperature, pore pressure and gas 


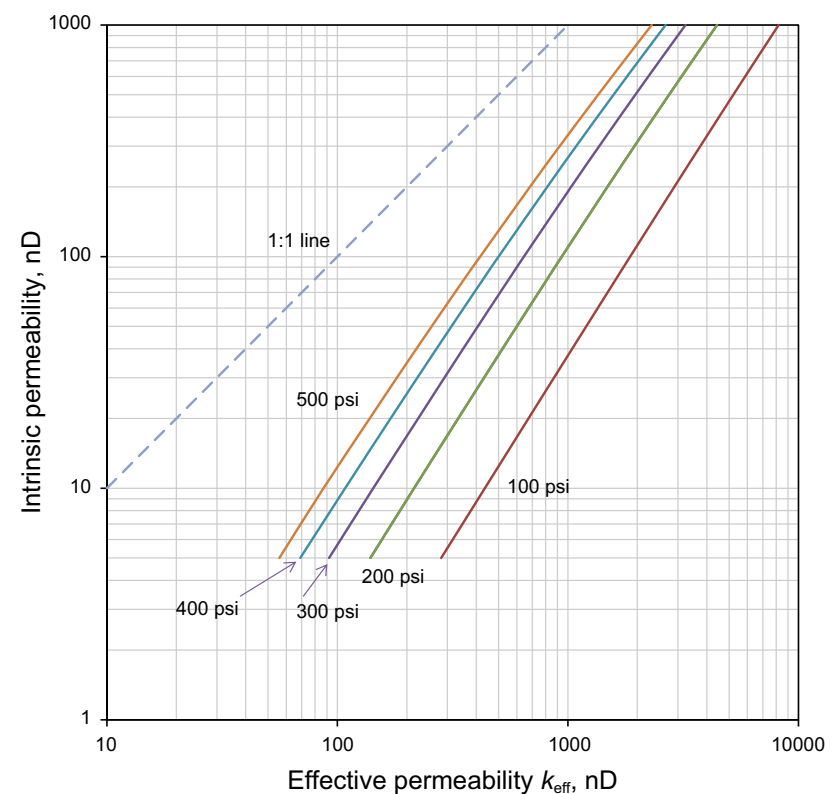

Fig. 9 Relation between measured gas permeability $k_{\text {eff }}$ and the intrinsic permeability at the room temperature $\left(22{ }^{\circ} \mathrm{C}\right)$

properties including the collision diameter (Eq. 2). As previously indicated, our discussion in this paper, for simplicity, is limited to cases in which effective stress is constant. The methodology developed herein, however, is not limited to the constant effective-stress condition. For variable effective-stress conditions, reservoir effective permeability is still equal to the intrinsic permeability multiplied by the correction factor, but the intrinsic permeability changes with effective stress (Liu 2017) and the correction factor is calculated by considering this change through Eq. (11) that is used to approximate pore radius from intrinsic permeability and porosity under the current effective stress.

\section{Concluding remarks}

Source-rock permeability is a key parameter controlling the gas production rate because it is the limiting factor for gas flow from the matrix to the production well. Measured permeability in the laboratory, however, is an apparent parameter that depends on pore pressure and temperature as a result of the relative importance of slip flow and diffusion in gas flow in low-permeability media. To estimate the intrinsic permeability, this paper presents a simple approach to correct the laboratory permeability measurements based on the theory for gas flow in a nano/microtube that includes effects of viscous flow, slip flow and Knudsen diffusion under different pore pressure and temperature conditions. Comparisons between the calculated results with our approach and the published laboratory data support the usefulness of the approach. The "corrected" (or intrinsic) permeability is considerably smaller than the measured permeability. A larger measured permeability generally corresponds to a smaller relative difference between measured and corrected permeability values. A plot based on the approach is also presented to describe the relationships between corrected and measured permeability for typical GRI permeability test conditions.

With the workflow presented in this study including both permeability correction and a relationship between measured permeability, laboratory test conditions and intrinsic permeability, one can relatively easily estimate the intrinsic permeability from laboratory data and calculate the effective permeability under the reservoir conditions using the intrinsic permeability and the relationship. It is the effective permeability corresponding to the reservoir conditions that should be used in reservoir simulators that describe the gas flow in source rocks with Darcy's law.

Acknowledgements We thank the management of Aramco Research Center and Saudi Aramco for the permission to publish this paper.

Open Access This article is distributed under the terms of the Creative Commons Attribution 4.0 International License (http://crea tivecommons.org/licenses/by/4.0/), which permits unrestricted use, distribution, and reproduction in any medium, provided you give appropriate credit to the original author(s) and the source, provide a link to the Creative Commons license, and indicate if changes were made.

\section{References}

Alnoaimi KR, Kovscek AR. Experimental and numerical analysis of gas transport in shale including the role of sorption. In: SPE annual technical conference and exhibition held, 30 September2 October, New Orleans, Louisiana, USA; 2013. https://doi.org/ 10.2118/166375-MS.

Beskok A, Karniadakis EG. A model for flows in channels, pipes, and ducts at micro and nano scales. Microscale Thermophys Eng. 1999;3(1):43-77. https://doi.org/10.1080/108939599199864.

Civan F. Effective correlation of apparent gas permeability in tight porous media. Transp Porous Med. 2010;82(2):375-84. https:// doi.org/10.1007/s11242-009-9432-z.

Civan F, Rai CS, Hondergeld CH. Determining shale permeability to gas by simultaneous analysis of various pressure tests. SPE J. 2012;17(3):717-26. https://doi.org/10.2118/144253-PA.

Cui X, Bustin AMM, Bustin RM. Measurements of gas permeability and diffusivity of tight reservoir rocks: different approaches and their applications. Geofluids. 2009;9(3):208-23. https://doi.org/ 10.1111/j.1468-8123.2009.00244.x.

Darabi H, Ettehad A, Javadpour F. Gas flow in ultra-tight shale strata. J Fluid Mech. 2012;710:641-58. https://doi.org/10.1017/jfm. 2012.424.

Hakami A, Al-Mubarak A, Al-Ramadan K, Kurison C, Poveda IL. Characterization of carbonate mudrocks of the Jurassic Tuwaig Mountain formation, Jafurah basin, Saudi Arabia: implications for unconventional reservoir potential evaluation. J Nat Gas Sci Eng. 2016;33:1149-68. https://doi.org/10.1016/j.jngse.2016.04. 009. 
Heller R, Vermylen J, Zoback M. Experimental investigation of matrix permeability of gas shales. AAPG Bull. 2014;98(5):975-95. https://doi.org/10.1306/09231313023.

Javadpour F. Nanopore and apparent permeability of gas flow in mudrock (shales and siltstone). J Can Pet Technol. 2009;48(8):16-21. https://doi.org/10.2118/09-08-16-DA.

Jin G, Perez HG, Agrawal G, et al. The impact of gas adsorption and composition on unconventional shale permeability measurement. In: SPE Middle east oil \& gas show and conference, 8-11 March, Manama, Bahrain, 2015. https://doi.org/10.2118/172744MS

Jones SC. A technique for fast pulse-decay permeability measurements in tight rocks. SPE Form Eval. 1997;12(1):19-25. https:// doi.org/10.2118/28450-PA.

Myron BA, Eli LI. Numerical analysis for applied science. Hoboken: Willey; 1998.

Liu HH. Fluid flow in the subsurface: history, generalization and applications of physical laws. Springer; 2017. ISBN: 978-3-31943448-3.
Liu QX, Cai ZY. Study on the characteristics of gas molecular mean free path in nanopores by molecular dynamics simulations. Int J Mol Sci. 2014;15(7):12714-30. https://doi.org/10.3390/ ijms150712714.

Ozkan E, Brown M, Raghavan R, et al. Comparison of fracturedhorizontal-well performance in tight sand and shale reservoirs. SPE Reserv Eval Eng. 2011;14(2):248-59. https://doi.org/10. 2118/121290-PA.

Roy S, Raju R, Chung HF. Modeling gas flow through micro-channels and nano pores. J Appl Phys. 2003;93(8):4870-9. https://doi.org/ 10.1063/1.1559936.

Singh H, Javadpour HSF, Ettehadtavakkol A, Darabi H. Nonempirical apparent permeability of shale. SPE Reserv Eval Eng. 2014;17(3):414-23. https://doi.org/10.2118/170243-PA.

Ziarani SA, Aguilera R. Knudsen's permeability correction for tight porous media. Transp Porous Media. 2012;91(1):239-60. https:// doi.org/10.1007/s11242-011-9842-6. 